\title{
Integration of metabolomics in heart disease and diabetes research: current achievements and future outlook.
}

\section{Abstract}

Metabolomics is an emerging and powerful discipline that provides an accurate and dynamic picture of the phenotype of mammalian systems through the study of endogenous and exogenous metabolites in cells, tissues, culture supernatants as well as biofluids. In the last 5 years an increase in the number of metabolomic investigations of cardiovascular diseases and diabetes has been observed. In this article the experimental strategies applied and recent examples of their application in disease and drug efficacy/toxicity biomarker detection and the employment for the discovery of new molecular pathophysiological processes related to disease onset and progression, as well as their usefulness in drug efficacy/toxicity, will be reviewed. An outlook of the requirements for future successes will also be discussed. 